\title{
TABOO VERSUS AXIOM
}

\section{KATUZI ONO}

Some important formal systems are really developable from a finite number of axioms in the lower classical predicate logic LK or in the intuitionistic predicate logic LJ. Any system of this kind can be developed in LK (or in LJ) from the single conjunction of all the axioms of the system.

I have been intending to develop usual formal systems starting from TABOOS and standing on the primitive logic LO at first, since the logic too could be brought up to the usual logics by means of TABOOS in LO ${ }^{1)}$. In developing any formal system from TABOOS, we are forced to assume unwillingly that all the TABOOS are mutually equivalent, as far as we adopt more than one TABOOS for the system. If we could develop formal systems from singleTABOO TABOO-systems, we could get rid of this unwilling assumption.

It would be well expected that any formal theory developable from a finite number of axioms in $\mathbf{L K}$ or in $\mathbf{L J}$ would be developable from a single TABOO. ${ }^{2}$ In the present paper, I will prove that this is really the case.

Before stating the theorem, let us define the $\hat{\xi}$-TRANSFORM $@[\xi]$ of any sentence $\Subset$ with respect to an $h$-ary relation $\mathfrak{F}(h \geq 0)$. $\subseteq[\hat{\jmath}]$ is introduced by a structural recursive definition as follows :

$$
\begin{aligned}
& \subseteq[\mathfrak{F}] \equiv(\tau)((\subseteq \rightarrow \mathfrak{W}(\tau)) \rightarrow \mathfrak{F}(\tau)) \text { for any elementary sentence } \Subset \text {, }
\end{aligned}
$$

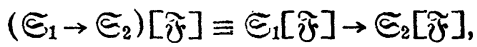

$$
\begin{aligned}
& ((x) \Im)[\mathfrak{F}] \equiv(x)(\Subset[\mathfrak{F}]) \text {, } \\
& \left(\Im_{1} \wedge \Im_{2}\right)[\hat{\jmath}] \equiv(\tau)\left(\left(\Im_{1}[\hat{\jmath}] \rightarrow\left(\mathfrak{\Im}_{2}[\hat{\mathfrak{F}}] \rightarrow \mathfrak{F}(\tau)\right)\right) \rightarrow \mathfrak{F}(\tau)\right),
\end{aligned}
$$

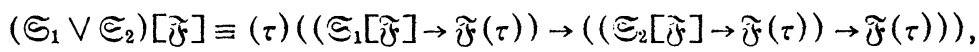

$$
\begin{aligned}
& (\rightarrow \Subset)[\hat{\jmath}] \equiv €[\hat{\jmath}] \rightarrow(\tau) \hat{\jmath}(\tau) \text {, }
\end{aligned}
$$

Received January 20, 1966.

1) As for this plan, see Ono [1]. LO is called PRIMITIVE SYSTEM OF POSITIVE LOGIC in Ono [1]. The terminology PRIMITIVE LOGIC together with its reference notation LO has been introduced in Ono [2].

2) In my work [1], I had to assume this to bring up logics by means of TABOO systems. The strong point of single-TABOO TABOO-systems has been pointed out also in Ono [3]. 


$$
((\exists x) \cong)[\mathfrak{F}] \equiv(\tau)((x)(\subseteq[\mathfrak{F}] \rightarrow \mathfrak{F}(\tau)) \rightarrow \mathfrak{F}(\tau)),
$$

where $\tau$ denotes a sequence of $h$ mutually distinct bound variables. $\tilde{F}$-transforms are expressible in terms of implication and universal quantification only as far as the relation $\tilde{\mho}$ is so.

According to the results of Ono [2] and [4], we have:

Lemma 1. Any sentence $\mathfrak{S}$ is provable in the logic LK if and only if $\mathfrak{S}[F]$ is provable in the logic LO for any proposition symbol $F$ (i.e. 0-ary relation symbol F) which is new for S. Any sentence expressible in terms of a single primitive notion $F$ (h-ary relation) is provable in the logic $\mathbf{L J}$ if and only if $\subseteq[F]$ is provable in the logic LO.

It should be noticed that we can assume without losing generality that any formal system developable from a finite number of axioms can be regarded as having only one primitive notion. For, any formal system developable from a finite number of axioms has only a finite number of primitive notions, say $R_{1}, \ldots, R_{m}$, where $R_{i}$ is an $n(i)$-ary relation for $i=1, \ldots, m$.

Let us now define $s(i)$ by

$$
s(i)=n(1)+\cdots+n(i-1) \quad(i=1, \ldots, m+1),
$$

and let us take up an $s(m+1)$-ary relation symbol $R$. Then, $R_{i}\left(x_{s\{i+1}, \ldots, x_{s(i+1)}\right)$ can be regarded as denoting

$$
\left(x_{1}\right) \cdots\left(x_{s(i)}\right)\left(x_{s(i+1)+1}\right) \cdots\left(x_{s(m+1)}\right) R\left(x_{1}, \ldots, x_{s(m+1)}\right) .
$$

(As for details, , see Ono [3].)

Before stating the main theorem, I will state two more lemmas concerning F-transforms.

Lemma 2. For any sentence $\Subset$ and for any relation $\mathfrak{F}$, say $h$-ary, $\subseteq(\mathfrak{F})$ is logically equivalent to

$$
(\tau)((\subseteq[\tilde{\mho}] \rightarrow \tilde{F}(\tau)) \rightarrow \mathfrak{F}(\tau))
$$

in the logic LO, where $\tau$ denotes a sequence of $h$ mutually distinct bound variables. $(h \geq 0$.)

This lemma can be proved by structural induction according to the definition of $\leqq[\hat{F}]$. As for detailed proof, see Ono [2]. 
Lemma 3. It is provable in LK that $\subseteq[;]$ is equivalent to $\subseteq$ for any contradictory proposition $\mathfrak{F}$. It is provable in $\mathbf{L} \mathbf{J}$ that $\subseteq[R]$ is equivalent to $\Xi$ for any relation $R$ and any sentence $\subseteq$ expressible in terms of $R$ only.

As for detailed proof, see Ono [4].

Main theorem. Let $\Sigma$ be any formal system developable from a single axiom $\mathfrak{A}$ in the logic LK. Then, any sentence $\subseteq$ is provable in $\Sigma$ if and only if $\Subset[\mathfrak{\mho}]$ is provable in the logic LO for the proposition $\mathfrak{F}$ defined by

$$
\mathfrak{F} ₹ \mathfrak{U}[R] \rightarrow R
$$

for any new proposition symbol $R$ for $\Sigma$.

Next, let $\Sigma$ be any formal system having $h$-ary relation $R$ as the sole primitive notion and developable from a single axiom $\mathfrak{P}$ in the logic $\mathbf{L J}$. Then, any sentence $\Subset$ is provable in $\Sigma$ if and only if $\widetilde{S}[\Im]$ is provable in the logic LO for the h-ary relation $\tilde{f}$ defined by

$$
\mathfrak{F}(\tau) \rightleftharpoons \mathfrak{Q}[R] \rightarrow R(\tau),
$$

where $\tau$ denotes a sequence of $h$ mutually distinct variables.

Proof. At first, I will discuss the case where $\Sigma$ is a formal system developable from the axiom $\mathfrak{A}$ in the logic $\mathbf{L K}$.

Let $\varsigma$ be any provable sentence in $\Sigma$, and $R$ be any proposition symbol which is new for $\Sigma$. Then, $\mathfrak{U} \rightarrow \mathbb{S}$ must be provable in LK. Accordingly, by

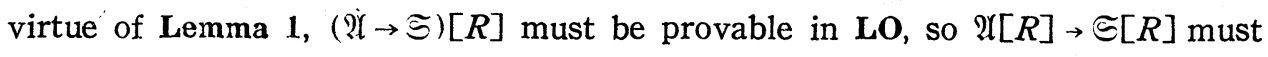
be also provable in LO by definition of $R$-transforms.

Now, let $\mathfrak{F}$ be the proposition defined by $\mathfrak{F} ₹ \mathfrak{\mathfrak { Y }}[R] \rightarrow R$. If we assume $\mathfrak{Y}[R]$, $R$ and $\tilde{f}$ turn out to be equivalent in Lo. Hence, $\mathfrak{A}[R] \rightarrow \subseteq[\Re]$ must be also provable in LO. According to Lemma 2, $\subseteq[\tilde{F}]$ is logically equivalent to

$$
(\subseteq[\mathfrak{F}] \rightarrow \mathfrak{F}) \rightarrow \mathfrak{F} \text { i.e },(\Subset[\tilde{\mathfrak{F}}] \rightarrow \mathfrak{F}) \rightarrow(\mathfrak{K}[R] \rightarrow R)
$$

in LO. However, the last sentence is surely equivalent to

$$
\mathfrak{H}[R] \rightarrow((\mathfrak{\subseteq}[\hat{\mathfrak{F}}] \rightarrow \mathfrak{W}) \rightarrow(\mathfrak{U}[R] \rightarrow R))
$$

i.e. to the sentence

$$
\mathfrak{U}[R] \rightarrow((\cong[\mathfrak{\mho}] \rightarrow \mathfrak{F}) \rightarrow \mathfrak{F}),
$$

which is deducible from $\mathfrak{X}[R] \rightarrow S_{[}[\hat{j}]$ in LO. Accordingly, we can see that 
$\subseteq[$; $]$ itself is provable in LO.

Conversely, let $R$ be a new proposition symbol for $\Sigma$, $\widetilde{\mho}$ be the proposition defined by $\mathfrak{F} ₹ \mathfrak{Y}[R] \rightarrow R$, and $€[\mathfrak{F}]$ be logically provable in LO. Then, $€[\mathfrak{F}]$ must be provable in LK too, because $\mathbf{L K}$ is a logic stronger than LO. Since $R$ is a new proposition symbol for $\Sigma$, the sentence $\subseteq[\tilde{\mho}]$ must be provable in LK even when we replace $R$ by any contradictory proposition $§, e . g$. the proposition $\mathfrak{C}$ defined by $\mathfrak{C} ₹ \mathfrak{B} \wedge \rightarrow \mathfrak{B}$ for any fixed proposition $\mathfrak{B}$.

For any sentence $\mathfrak{I}$, let us denote by $\mathfrak{I}^{*}$ the sentence obtained from $\mathfrak{I}$ on replacing all the proposition symbols $R$ in $\mathfrak{I}$ by the contradictory proposition ๒. Then, $\subseteq[\mathfrak{F}]^{*}$ i.e. $\subseteq\left[\mathfrak{F}^{*}\right]$ is logically provable in LK. $\mathfrak{U}[R]^{*}$ i.e. $\mathfrak{U}[\mathfrak{\complement}]$ can be proved equivalent to $\mathfrak{H}$ by Lemma 3. Moreover, if we assume $\mathfrak{A}$ i.e. $\mathfrak{U}[\complement]$,

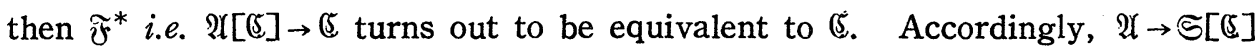
must be logically provable in LK. Since $₫$ is a contradictory proposition, $\Subset[\Subset]$ is logically equivalent to $\subseteq$ in $\mathbf{L K}$ according to Lemma 3. Hence, $\mathfrak{U} \rightarrow \subseteq$ is provable in LK. Since $\Sigma$ is assumed to be developable from the sole axiom $\mathfrak{A}$ standing on the logic $\mathbf{L K}, \subseteq$ is provable in the formal system $\Sigma$.

Next, I will discuss the case where $\Sigma$ is a formal system having $h$-ary relation $R$ as the sole primitive notion and developable from a single axiom $\mathfrak{l}$ in the logic LJ. Also in this case, we can carry out the proof almost in parallel with the foregoing case.

Namely, let $\subseteq$ be any provable sentence in $\Sigma$. Then, $(\mathfrak{A} \rightarrow \Im)[R]$ must be provable in LO. Since $(\mathfrak{H} \rightarrow \subseteq)[R]$ denotes $\mathfrak{X}[R] \rightarrow \subseteq[R]$, we can see that $\mathfrak{A}[R] \rightarrow \Subset[R]$ is provable in LO.

Now, let the $h$-ary relation $\tilde{f}$ be defined by

$$
\mathfrak{\mho}(\tau) ₹ \mathfrak{Y}[R] \rightarrow R(\tau),
$$

where $\tau$ denotes any sequence of $h$ mutually distinct variables. If we assume $\mathfrak{V}[R]$, then $\mathfrak{F}$ and $R$ turn out to be mutually equivalent relations in the sense that $(\tau)(\mathfrak{F}(\tau) \equiv R(\tau))$ holds in LJ. Hence, $\mathfrak{X}[R] \rightarrow \subseteq[\widetilde{\Im}]$ must be provable in LO.

According to Lemma 2, $\subseteq[\tilde{\mho}]$ is equivalent to $(\tau)((\subseteq[\tilde{\mho}] \rightarrow \widetilde{F}(\tau)) \rightarrow \hat{\jmath}(\tau))$, so

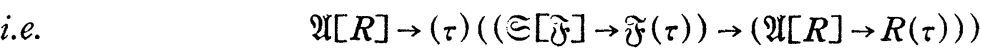


must be provable in LO.

The last sentence can be proved equivalent to

$$
(\tau)((\subseteq[\hat{\mho}] \rightarrow \mathfrak{\mho}(\tau)) \rightarrow(\mathfrak{U}[R] \rightarrow R(\tau))),
$$

which is equivalent to $\Subset[\mathfrak{F}]$ according to Lemma 2. So, $\Subset[\mathfrak{F}]$ is provable in LO.

Conversely, let $\subseteq$ be any sentence of the formal system $\Sigma$ for which $\Subset[₹]$ is provable in LO. Then, $€[\tilde{\mho}]$ must be provable in $\mathbf{L J}$, since $\mathbf{L J}$ is a logic stronger than LO.

If we assume $\mathfrak{U}$, we can prove $\mathfrak{H}[R]$ in $\mathbf{L J}$ by virtue of Lemma 3 , so the relation $\widetilde{f}$ can be proved equivalent to the relation $R$ by definition of the relation $\mathfrak{F}$. Hence, $\subseteq[R]$ is deducible in $\mathbf{L J}$ from the assumption $\mathfrak{H}$. Because $\S[R]$ can be proved equivalent to $\S$ in $\mathbf{L J}$ by virtue of Lemma 3, we can see that $\subseteq$ is deducible from $\mathfrak{A}$ in the logic LJ. Since the formal system $\Sigma$ is developable from the single axiom $\mathfrak{U}$ in the logic $\mathbf{L J}$, the sentence $\Subset$ is provable in $\Sigma$.

Concluding remark. If we can only state the axiom system of any formal system standing on the logic $\mathbf{L K}$ or $\mathbf{L J}$, we can develop the system in the primitive logic LO starting from a single TABOO by virtue of the main theorem. Especially, we can establish either of the logics $\mathbf{L K}$ and $\mathbf{L J}$ itself from a TABOO as a special case of formal systems standing on $\mathbf{L K}$ or $\mathbf{L J}$.

For formal systems $\Sigma$ developable from axiom schemes which may contain unlimited number of axioms, we can only say that any concrete theory in $\Sigma$ can be established from a single TABOO (depending on each theory), since any concrete theory would be developable from a finite number of axioms.

\section{REFERENCES}

[1] Ono, K., [1] A certain kind of formal theories, Nagoya Math. J., 25 (1965), 59-86.

[2] Ono, K., [2] On universal character of the primitive logic, Nagoya Math. J., 27-1 (1966), $331-353$

[3] Ono, K., +[3] On development of formal systems starting from primitive logic, Nagoya Math. J., 28 (1966), 79-83.

[4] Ono, K., [4] Formal system having just one primitive notion, Nagoya Math. J., 28 (1966), 73-77.

Mathematical Institute, Nagoya University 